\title{
Bringing homeless deaths to light
}

\author{
Cite as: CMAJ 2017 March 20;189:E446-7. doi: 10.1503/cmaj.1095399
}

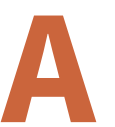
fter dinner in the basement hall of St. Patrick's Church in central Toronto, volunteers with Out of the Cold begin clearing the concrete floor so sleeping mats can be laid out. Some 80 or so homeless people will be staying overnight.

One of them is 54-year-old Angela, who lost her home six years ago during a period of severe mental instability. She pushes back her dinner plate wearily and reaches for her cane. It's been snowing heavily all day and she was out in the storm until the shelter opened at $4 \mathrm{pm}$. "Even though I suffer from diabetes, epilepsy, schizophrenia and depression," she says, "I've been on the city's housing wait list for three years now."

With 176000 others also on that list, Angela says she expects to be homeless for a long time to come. And that means her chances of living out her natural lifespan could be reduced by as much as $40 \%$, according to a study by Dr. Stephen Hwang, director of the Centre for Urban Health Solutions at Toronto's St. Michael's Hospital.

But as revealing as this figure is, says Hwang, "far too little is known about the true extent and causes of mortality among the homeless in Canada."

On any given night, 35000 Canadians are homeless, including some 5000 in Toronto.

Joyce Bernstein, an epidemiologist and statistician with Toronto Public Health Surveillance and Epidemiology Unit, persuaded Toronto's city council to conduct rigorous surveillance of mortality among the homeless. Data are being collected by the Homelessness and Mortality Research \& Advocacy Collaborative, which includes five community service agencies, researchers and public health officials.

"There are so many more deaths as compared to the general population,"

says Bernstein, who in 2012 mounted a city-wide surveillance effort that recruited more than 30 agencies. This showed that more than 300 babies are born among Toronto's homeless annually. "We have no clear idea how many homeless people are dying."

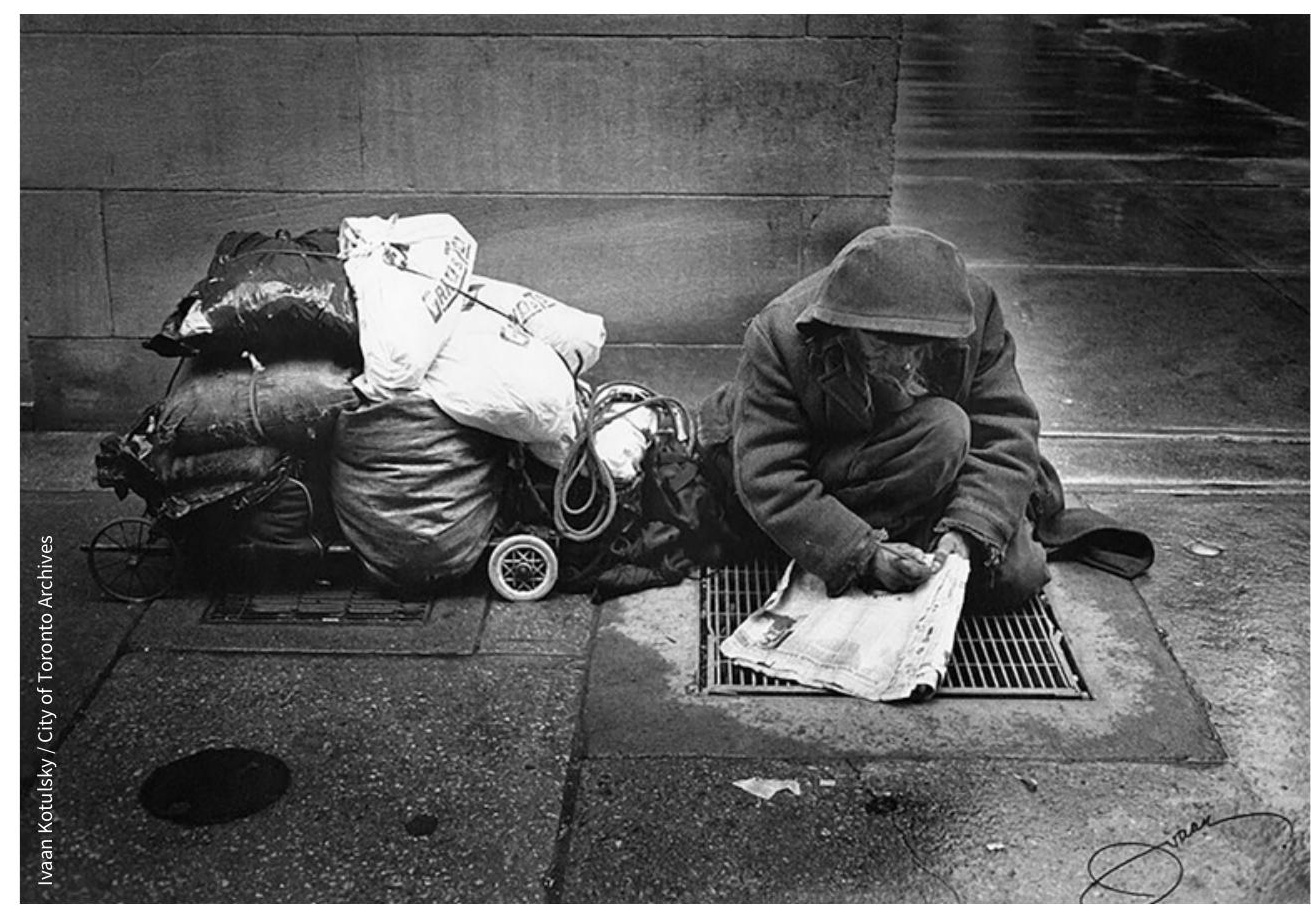

Homelessness can reduce life expectancy by as much as $40 \%$.

"It's a huge win that they are at last counting deaths in Toronto," says Brian Davis, executive director of Houselink, a Toronto agency that finds housing for homeless people with mental illnesses.

Bernstein provides a glimpse of early, unpublished data on 119 homeless people who died between March 2013 and October 2016 on downtown Toronto's west side. Many had multiple causes, but some were clear cut. For example, 12 died of drug overdoses, and four were murdered or died after being assaulted (including one case described as a police assault). Thirty-one died of unknown causes. Only 16 of the 119 were older than 60; 34 were Indigenous people.

Alcohol and substance abuse, or related disorders such as cirrhosis, are cited in 47 cases. Fifty-six had some type of housing and 35 reportedly had health cards - although that may be an under- estimate due to some inconsistencies in data collection.

Lorie Steer, director of housing and homeless services at St. Stephen's Community House, one of the five agencies involved in the data gathering, says the original decision to begin tracking deaths among the homeless came "after a sharp increase in premature and preventable deaths was noted, especially among homeless indigenous people."

Steer notes that if a death is considered "natural," and the coroner is not involved, "we may never know the cause of death. So a 25-year-old who dies of 
"organ failure" - likely related to high levels of alcohol use - in hospital would have the cause of death listed as natural. Would we really consider this a natural death?"

Historically, "homeless deaths were often talked about in the context of death on the street from exposure," says Steer. "There was a sense that street homelessness, combined with a lack of access to health services and affordable housing contributed to these deaths. But many of the individuals we knew of who were dying had subsidized housing, sometimes supportive housing. The number of deaths, the often young age, and tragic stories associated with these deaths, all need to be brought to light."

Paul Webster, Toronto, Ontario 